\title{
Measurement of the cross-section of the associated production of a top quark pair and a $W$ or $Z$ boson with the ATLAS detector
}

\author{
Sebastian Heer*† \\ On behalf of the ATLAS Collaboration \\ Physikalisches Institut, Universität Bonn, Germany \\ E-mail: heerduni-bonn. de
}

\begin{abstract}
In the Standard Model the coupling of the top quark to a vector boson is predicted via the weak interaction. New physics beyond the Standard Model can modify the $t \bar{t} V$ cross-section. As an example several of the operators introduced by effective field theory are sensitive to the $t \bar{t} Z$ and $t \bar{t} W$ production cross-sections. Multiple analysis channels with 2, 3 or 4 lepton final-states have been considered in the latest $t \bar{t} V$ cross section measurement by the ATLAS experiment located at the Large Hadron Collider (LHC). The dominant backgrounds in the analysis are mis-reconstructed leptons and the diboson process, depending on the analysis channel. A two-dimensional fit of the $t \bar{t} W$ and $t \bar{t} Z$ cross-section is performed to interpret possible deviations from the Standard Model.
\end{abstract}

An Alpine LHC Physics Summit (ALPS2018)

15-20 April, 2018

Obergurgl, Austria

\footnotetext{
*Speaker.

${ }^{\dagger}$ Supported by European Research Council grant ERC-CoG-617185
} 


\section{Introduction}

The latest published $t \bar{t} V$ analysis by ATLAS [1] analyzed the $3.2 \mathrm{fb}^{1}$ dataset taken in 2015 [2]. The aim is a cross section measurement of both $t \bar{t} Z$ and $t \bar{t} W$ by simultaneously fitting both in a profile likelihood fit. Multiple analysis channels are considered, which are introduced in Section 2. Both processes are predicted by the Standard Model and a measurement of the cross section can indicate new physics, which could be interpreted with various models, such as effective field theories (EFT) which modify the SM Lagrangian, for example in Reference [6]. A previous measurement by ATLAS analyzed the $\sqrt{s}=8 \mathrm{TeV}$ dataset and was in agreement with the Standard Model [3] and a more recent measurement by the CMS experiment can be seen in Reference [5].

\section{Analysis overview and event selection}

The $t \bar{t} V$ analysis is split into multiple analysis channels which are sensitive to either $t \bar{t} W, t \bar{t} Z$ or both. The main backgrounds include fake leptons, which is estimated by the data-driven matrix method for the di- and trilepton channels and by a MC based method for the tetralepton channels, and the diboson processes.

Only events with at least two leptons are considered, of which at least one lepton is required to satisfy $p_{\mathrm{T}}>25 \mathrm{GeV}$. Detailed event selection for each region are described below.

\section{$2.1 t \bar{t} W$ analysis}

The $t \bar{t} W$ analysis is performed in the same-sign dimuoun channel ( $2 \mu \mathrm{SS})$ and one of the trilepton $t \bar{t} Z$ regions, that also provides some sensitivity to $t \bar{t} W$. This channel provides the highest sensitivity among all channels, due to the high misidentifaction rate of electrons. Events are required to have two muon candidates, with the same charges and $p_{\mathrm{T}}>25 \mathrm{GeV}, E_{\mathrm{T}}^{\text {miss }}>40 \mathrm{GeV}$, the scalar sum of the $p_{\mathrm{T}}$ of the selected leptons and jets, $H_{\mathrm{T}}$, above $240 \mathrm{GeV}$, and at least two $b$-tagged jets.

The dominant background in the $2 \mu \mathrm{SS}$ channel arises from events containing fake leptons, where the main source is $t \bar{t}$ events. A total of 9 events have been observed in the $2 \mu \mathrm{SS}$ channel.

\section{$2.2 t \bar{t} Z$ analysis}

\section{Trilepton analysis}

Four signal regions with exactly three leptons are considered. The first three are sensitive to $t \bar{t} Z$; each of these requires an opposite-sign same-flavour (OSSF) pair of leptons whose invariant mass is within $10 \mathrm{GeV}$ of the $Z$ boson mass. The signal regions are categorized by their jet and $b$-jet multiplicities and have different signal-to-background ratios. In the $3 \ell-Z-1 \mathrm{~b} 4 \mathrm{j}$ region, at least four jets are required, exactly one of which is $b$-tagged. In the $3 \ell-Z-2 \mathrm{~b} 3 \mathrm{j}$ region, exactly three jets with at least two $b$-tagged jets are required. In the $3 \ell-Z-2 \mathrm{~b} 4 \mathrm{j}$ region, at least four jets are required, of which at least two are $b$-tagged.

In the $3 \ell$-noZ- $2 b$ region at least two and at most four jets are required, of which at least two are $b$-tagged, no OSSF lepton pair is allowed in the $Z$ boson mass window, and the sum of the lepton charges must be \pm 1 . This region primarily targets the $t \bar{t} W$ process but also has a sizable $t \bar{t} Z$ contribution. 
The main backgrounds are the $W Z$ process and fake leptons for the trilepton analysis. A total of 29 events have been observed among the four trilepton regions.

\section{Tetralepton analysis}

Four signal regions with exactly four leptons are considered. Events with two pairs of oppositesign leptons are selected, and at least one pair must be of same flavour. The OSSF lepton pair with reconstructed invariant mass closest to $m_{Z}$ is attributed to the $Z$ boson decay and denoted $Z_{1}$. The two remaining leptons are used to define $Z_{2}$. The signal regions are defined according to the relative flavour of the two $Z_{2}$ leptons, different flavour (DF) or same flavour (SF), and the number of $b$-tagged jets: one, or at least two $(1 b, 2 b)$. The signal regions are thus $4 \ell$-DF- $1 \mathrm{~b}, 4 \ell$-DF- $2 \mathrm{~b}$, $4 \ell$-SF- $1 b$ and $4 \ell$-SF- $2 b$.

To suppress events with fake leptons in the 1-b-tag multiplicity regions, additional requirements on the scalar sum of the transverse momenta of the third and fourth leptons $\left(p_{\mathrm{T} 34}\right)$ are imposed. In the $4 \ell-\mathrm{SF}-1 \mathrm{~b}$ and $4 \ell$-DF- $1 \mathrm{~b}$ regions, events are required to satisfy $p_{\mathrm{T} 34}>25 \mathrm{GeV}$ and $p_{\mathrm{T} 34}>35 \mathrm{GeV}$, respectively. In all regions, the invariant mass of any two reconstructed OS leptons is required to be larger than $10 \mathrm{GeV}$.

The main backgrounds are the $Z Z$ process, primarily in the SF regions, and fake leptons. A total of 5 events have been observed among the four tetralepton regions.

\section{Results}

A total of 43 events have been observed among all signal regions. Table 1 shows a list of uncertainties with their impact on the measurement for both $t \bar{t} Z$ and $t \bar{t} W$. As can be seen, both measurements are dominated by the statistical uncertainty. Figure 1 shows the extracted cross sections of the simultaneous fit of $t \bar{t} Z$ and $t \bar{t} W$, compared to the Standard Model prediction.

\begin{tabular}{lcc}
\hline Uncertainty & $\sigma_{t \bar{t} Z}$ & $\sigma_{t \bar{t} W}$ \\
\hline Luminosity & $2.6 \%$ & $3.1 \%$ \\
Reconstructed objects & $8.3 \%$ & $9.3 \%$ \\
Backgrounds from simulation & $5.3 \%$ & $3.1 \%$ \\
Fake leptons and charged misID & $3.0 \%$ & $19 \%$ \\
Signal modelling & $2.3 \%$ & $4.2 \%$ \\
\hline Total systematic & $11 \%$ & $22 \%$ \\
Statistical & $31 \%$ & $48 \%$ \\
\hline Total & $32 \%$ & $53 \%$ \\
\hline
\end{tabular}

Table 1: List of dominant and total uncertainties in the measured cross sections of the $t \bar{t} Z$ and $t \bar{t} W$ processes from the fit. All uncertainties are symmetrised [2].

The extracted cross sections are $\sigma_{t \bar{t} Z}=0.92 \pm 0.29$ (stat.) \pm 0.10 (syst.) pb and $\sigma_{t \bar{t} W}=$ $1.50 \pm 0.72$ (stat.) \pm 0.33 (syst.) pb, which are both in agreement of the theory prediction of $\sigma_{t \bar{Z} Z}^{\mathrm{SM}}=0.84 \pm 0.09 \mathrm{pb}$ and $\sigma_{t \bar{t} W}^{\mathrm{SM}}=0.60 \pm 0.08 \mathrm{pb}[4]$. 


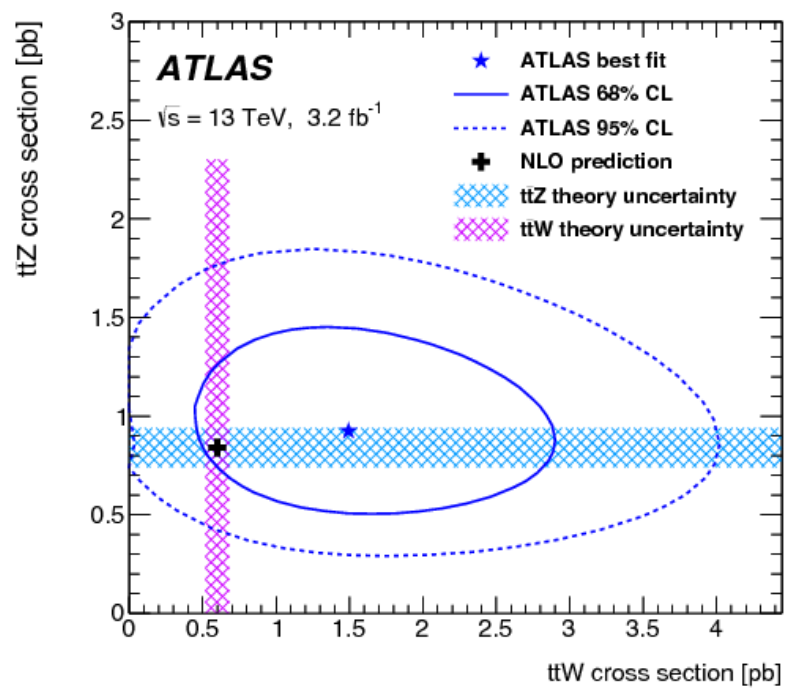

Figure 1: The result of the simultaneous fit to the $t \bar{t} Z$ and $t \bar{t} W$ cross sections along with the $68 \%$ and 95\% confidence level (CL) contours. The shaded areas correspond to the theoretical uncertainties in the Standard Model predictions, and include renormalisation and factorisation scale uncertainties as well as PDF uncertainties including $\alpha_{s}$ variations [2].

\section{Conclusions}

The measured cross sections using the 2015 dataset of $3.2 \mathrm{fb}^{-1}$ of $\sigma_{t \bar{t} Z}=0.92 \pm 0.29$ (stat.) \pm 0.10 (syst.) $\mathrm{pb}$ and $\sigma_{t \bar{W}}=1.50 \pm 0.72$ (stat.) \pm 0.33 (syst.) pb measured with the ATLAS experiment at the LHC are in agreement with the Standard Model prediction [4]. The uncertainties are dominated by the statistical uncertainty and the systematic uncertainties on the fake estimation. A measurement with more data and an improved fake estimation can significantly improve these results. It is interesting to study the shape of certain distributions, like the $p_{\mathrm{T}}$ or the $\Delta \phi$ of the leptons from the $Z$ boson. Those distributions should be sensitive to EFT operators [6] and can be studied with a larger dataset in the future.

\section{References}

[1] ATLAS Collaboration, The ATLAS Experiment at the CERN Large Hadron Collider, JINST 3 (2008) S08003

[2] ATLAS Collaboration, Measurement of the $t \bar{t} Z$ and $t \bar{t} W$ production cross sections in multilepton final states using $3.2 \mathrm{fb}^{1}$ of pp collisions at $\sqrt{\mathrm{s}}=13 \mathrm{TeV}$ with the ATLAS detector, Eur. Phys. J. C77 (2017) 40, arXiv:1609.01599 [hep-ex].

[3] ATLAS Collaboration, Measurement of the $t \bar{t} Z$ and $t \bar{t} W$ production cross sections in pp collisions at $\sqrt{s}=8 \mathrm{TeV}$ with the ATLAS detector, JHEP11 (2015) 172, arXiv:1509.05276 [hep-ex].

[4] J. Alwall, R. Frederix et al., The automated computation of tree-level and next-to-leading order differential cross sections, and their matching to parton shower simulations JHEP07 (2014) 079, arXiv:1405.0301 [hep-ph]. 
[5] CMS Collaboration, Measurement of the cross section for top quark pair production in association with a $W$ or Z boson in proton-proton collisions at $\sqrt{s}=13 \mathrm{TeV}$, CMS-TOP-17-005, arXiv:1711.02547 [hep-ex].

[6] R. Röntsch, M. Schulze, Probing top-Z dipole moments at the LHC and ILC, JHEP08 (2015) 044, arXiv:1501.05939 [hep-ph]. 